\title{
Consciousness Examined: An Introduction to the Foundations of Neurobiology for EMDR
}

\author{
Uri Bergmann \\ Commack, New York
}

\begin{abstract}
The human mind is difficult to investigate, but the biological foundations of the mind, especially consciousness, are generally regarded as the most daunting. In this article, excerpted from the book Neurobiological Foundations for EMDR Practice (Bergmann, 2012), we introduce and outline aspects of consciousness, information processing, and their relationship to eye movement desensitization and reprocessing (EMDR). We examine consciousness with respect to three characteristics: unity of perception and function, subjectivity, and prediction. The relationship of these characteristics to EMDR is examined.
\end{abstract}

Keywords: consciousness; information processing; prediction; EMDR

$\mathbf{T}$ he material in this article is an excerpt from the book Neurobiological Foundations for EMDR Practice. The book introduces the most current research concerning the neural underpinnings of consciousness and EMDR in regard to attachment, traumatic stress, and dissociation. It is the first book to comprehensively integrate new findings in information processing, consciousness, traumatic disorders of information processing, chronic trauma and autoimmune compromises, and the implications of these data on the adaptive information processing (AIP) model and EMDR treatment.

The book examines online/wakeful information processing, including sensation, perception, somatosensory integration, cognition, learning, memory, language and motricity, as well as off-line/sleep information processing, such as slow wave sleep and cognitive memorial processing, and REM/dream sleep and its function in semantic and emotional memory processing. The volume also addresses disorders of consciousness, including coma, anesthesia, and other neurological disorders, with particular focus on disorders of Type 1 PTSD, complex PTSD/dissociative disorders, and personality disorders. It delves into chronic trauma and autoimmune function, especially in regard to medically unexplained symptoms
(MUS), and examines them from the perspective of autoimmune compromises resulting from the unusual neuroendocrine profile of PTSD sufferers. The final section integrates all material to illustrate the tenets of the AIP model and the implication of this material with respect to current EMDR treatment, as well as techniques to render it more robust.

\section{Consciousness}

The human mind is difficult to investigate, but the biological foundations of the mind, especially consciousness, are generally regarded as the most daunting. Antonio Damasio (1999) has argued that if elucidating the nature of the mind is the last frontier of the life sciences, consciousness often seems like the last mystery in the illumination of the mind. He notes,

The matter of mind, in general, and of consciousness in particular, allows humans to exercise to the vanishing point, the desire for understanding and the appetite for wonderment at their own nature that Aristotle recognized as so distinctively human. What could be more difficult to know than to know how we know? What could be more dizzying than to realize that it is our having consciousness

Editor's Note: This article is a modified reprint from Neurobiological Foundations for EMDR Practice, by U. Bergmann, 2012, New York, NY: Springer Publishing. Copyright 2012 by Springer Publishing. Reprinted with permission. 
which makes possible and even inevitable our questions about consciousness? (p. 4)

Echoing this sentiment, Alan Hobson (2009) opines that "consciousness, we are relieved to admit, is finally a bona fide subject of inquiry. Let us take the first obvious step and teach it to study itself' (p. xi).

For Rodolfo Llinas (2001), consciousness is a function of mindness, driving him to ask,

Why is mindness so mysterious to us? Why has it always been this way? The processes that generate such states as thinking, consciousness, and dreaming are foreign to us, I fancy, because they always seem to be generated with no apparent relation to the external world. They seem impalpably internal. (p. 4)

Similarly, Alan Hobson (2009) observes

The brain still tends to keep most of its activity out of consciousness, but what it excludes or admits is governed more by rules of activation, neuromodulation, input-output gating than by the predominance of repression. The unconscious is now seen as a useful lookup system for the conscious brain rather than a seething source of devils aiming at the disruption of consciousness. Consciousness itself is, thus, a tool for the investigation of itself as well as for the study of that part of the unconscious that is dynamically repressed. (p. xi)

Throughout his writings, Sigmund Freud articulated his ideas through the organizing concepts of the "self" and the "object." For Freud, the people interacting with the self were the objects of the self s drives and desires. Ironically, neuroscientists today, tend to view consciousness, from its basic levels to its utmost complexity, as the integrated neural function that brings together the object and the self.

Accordingly, Damasio (1999) opines,

At its elemental and most basic level, consciousness lets us recognize an irresistible urge to stay alive and experience a concern for the self. At its most profound and elaborate level, consciousness helps us develop a concern for other selves and improve the art of life. (p. 5)

Evolution, over these millions of years has given rise to our complex brain and, somehow, through the interactions among its 100 billion neurons, connected by trillions of synapses, our conscious experience of the world and of ourselves emerges.

Like it or not, consciousness is the pivotal biological function that allows us to know sorrow and joy, suffering and pleasure, embarrassment and pride, and grief and reunion. Damasio (1999) muses, "Do not blame Eve for knowing; blame consciousness, and thank it, too" (p. 4).

\section{The Mystery of Consciousness}

How, then, does the brain's internal activity actually represent the external world? How does it allow us to interact with the external world and internally with our own? How can it manage to differentiate external from internal reality? If consciousness is intrinsic to brain function, then is there some sort of place within the brain that we can isolate as being the seat of consciousness, or do we have to look more for a neural network distributed over a larger scale, throughout the brain?

An alternative way of exploring these mysteries is to ask how the brain mediates information processing, taking us to the study of individual neurons and their relationship to neural systems. Individual neurons function together in specialized groups, or systems, each serving a specific function. Systems neuroscience is the study of these neural systems, which include those involved in vision, memory, language, emotion, and motor function. Accordingly, these systems possess common properties, particularly in that they all process high-order information regarding our environment and biological needs. Consequently, the study of systems neuroscience places paramount emphasis on the identification of the neural structures and events associated with the hierarchical steps in information processing. Hence, we can now refine our questions further and ask the following: How is information encoded (sensation)? How is it interpreted to confer meaning (perception)? How is it modified or stored (learning and memory)? How is it used to predict the future state of the environment and the consequences of action (decision making/emotion)? How is it used to guide behavior (motor control) and to communicate (language)?

\section{Consciousness and Evolution}

Eric Kandel (2006) has argued that every revolution has its origins in the past and that the revolution that culminated in the new science of mind is no exception. Although the central role of biology in the study of mental or psychological processes was new, the profound ability of biology to influence the way we see ourselves was not. In the mid-19th century, Charles Darwin argued that we were not uniquely created but rather evolved gradually from lower animal ancestors. He proposed the even more daring idea that evolution's driving force was not a 
conscious, intelligent, or divine purpose but a "blind" process of natural selection, a completely mechanistic sorting process of random trial and error (a tinkering) based on hereditary variations.

Darwin's ideas challenged the teaching of most religions. Because biology's original purpose had been to explain the divine design of nature, his ideas shredded the historic bond between religion and biology. Kandel (2006) notes that consequently, for some, the neurobiology of mind is potentially more disturbing because it suggests that not only the body but also mind and the specific molecules that underlie our highest mental processes, of consciousness of self and others and of the past and future, have evolved from our animal ancestors. Nonetheless, as we shall see, this evolutionary perspective has profoundly facilitated our understanding by guiding us to study the nervous systems of lower animals, such as the mollusk Aplysia. By studying learning, such as habituation, sensitization, and conditioning in this animal, the neural patterns of learning and memory were clearly and consistently codified for the first time. With time and increased sophistications in technology, similar neural patterns were discovered in human functioning, confirming both Darwin's ideas of evolutionary connectedness and Ramon y Cajal's (1899) theories of synaptic plasticity.

\section{Characteristics of Consciousness}

In the past 20 years, consciousness has also been described and investigated with respect to three characteristics: unity, subjectivity, and prediction.

Unity of Consciousness. The unitary nature of consciousness refers to the fact that our experiences come to us as a unified whole. All of the various sensory modalities (i.e., color, shape, depth, sound, taste, and smell) are integrated and melded into a single, coherent conscious experience.

Subjectivity. The foregoing speaks to information that comes to us from the outside world. The same, however, is also true of our inner, proprioceptive experiences, wherein modalities such as associations, memories, and emotions are likewise integrated and melded with our sensory experiences to produce a coherent subjective experience.

Prediction. Regarding prediction, it has been noted by Llinas (2001) that consciousness, or the mindness state, which may or may not represent external reality (subjective states, imagining or dreaming), has

evolved as the goal-oriented device that implements predicted/intentional interactions between a living organism and its environment.
Such transactions, to be successful, require an inherited, prewired instrument that generates an internal subjective image of the external world that can be compared with sensory transduced objective information from the external environment. (p. 3)

Given that this functional comparison of internally generated sensorimotor images, with real-time sensory information from the organism's immediate environment, is defined as perception, then the function of the underlying workings of perception is to mediate prediction, that is, "the useful expectation of events yet to come" (p. 3). From an evolutionary/ survival perspective, which is the driving force that all species have in common, prediction, with its goaloriented essence, is at the very core of brain function and consciousness. Obviously, the ability to predict is critical in the animal kingdom; a creature's life depends upon it. Still, the mechanism of prediction is far more ubiquitous in the brain's control of body functions.

\section{Prediction at Work}

Consider the simple act of reaching for a carton of milk in the refrigerator. Without giving much thought to our actions, we must predict the carton's weight, its slipperiness, its degree of fullness, and finally, the compensatory balance we must apply for a successful smooth trajectory of the contents of the carton into our glass.

The brain's predictive ability can also be generated in the absence of conscious awareness. As an example, have you ever found yourself blinking just before a bug lands in your eye? You did not observe the bug, at least on a conscious level, yet you, somehow, predicted the event and blinked appropriately to prevent its entry into your eye. Prediction is at the heart of this protective mechanism (Llinas, 2001).

Prediction, continually operative at conscious, unconscious, and reflexive levels, is pervasive throughout most if not all levels of brain function. As we shall see, in order for the nervous system to predict, it must perform a rapid comparison of the sensory properties/input of the external world with a separate internal/subjective representation of the world. In humans (and a number of animals), this internal representation is driven by sensorimotor experiences, associations, memories, and emotions. This precarious balance of outer objectivity and inner subjectivity and the predictions that it generates about ourselves, our environment, and those around us is at the heart of our knowledge, creativity, joys and sorrows, and the quality of our relationships. 


\section{Consciousness and EMDR}

Consciousness and EMDR have been intimately related, albeit under a different name. Whereas the field of neurobiology has utilized the term consciousness to denote the processes of sensation, perception, learning, cognition, emotion, somatosensory integration, and memory; the discipline of psychology has chosen to use the term information processing. Accordingly, they will be used interchangeably. If we tend to favor the term consciousness, it is only because it feels more human.

Throughout the past 20 years, EMDR has evolved into a therapeutic approach guided by the adaptive information processing (AIP) model (Shapiro, 2001). In 1990 , the name change from eye movement desensitization (EMD) to eye movement desensitization and reprocessing (EMDR) heralded a change in orientation from the initial behavioral formulation of simple desensitization of anxiety to a more integrated information processing paradigm. This evolution ushered in the accelerated information processing model, which illustrated a clinically grounded understanding of the underlying principles that govern perception and the integration of new information within cognitive, memorial, and emotional frameworks (Shapiro, 1995). In 2001, this continued evolution brought us the aforementioned adaptive information processing model. Regarding these models, Francine Shapiro (2001) has argued that "their utility lies in their ability not only to explain, but to predict clinical outcomes" (p. 14).

\section{Prediction and EMDR}

As we shall see, prediction and information processing also hold a crucial place in EMDR treatment, given the centrality of the negative and positive cognitions, both of which articulate predictions regarding the environment, others/objects, and the self. In view of that, a distorted negative cognition is clearly symptomatic of maladaptive predictive information processing.

Like other species of animals, we process information in order to generate predictions about the environment (safe, dangerous, poisonous, nutritious?) and about those around us (safe, trustworthy, dangerous?). However, we humans also generate predictions about ourselves (good, bad, able, powerless?).

In all these permutations of consciousness and information processing, oscillating neural systems, which correspond to the various outer sensory and inner proprioceptive modalities, are synchronized with respect to their oscillations to produce coherent (unified) conscious experiences-processes referred to as neural linkage and temporal binding.

\section{The Progress of Science}

Reflecting on the foregoing, it becomes apparent that the understanding of the human mind in biological terms has emerged as one of the most important challenges for science in the 21st century. Our goal in this endeavor has been to understand the biological underpinnings of sensation, perception, cognition, learning, memory, emotion, and sensory integration.

The progress that researchers have made in the field of neuroscience, unthinkable even a few decades ago, has made possible our present understanding. The discovery of the structure of DNA in 1953 revolutionized biology, giving it a foundational framework for comprehending the mechanisms underlying the gene's ability to control the functioning of cells. This breakthrough led to a basic understanding of gene regulation and gene-related cell function, propelling an understanding of the science of biology to a level rivaling that of physics and chemistry.

Imbued with this knowledge, biology turned its focus to its loftiest goal, the understanding of the biological nature of the human mind. This endeavor, once considered to be prescientific and impossible, has achieved great momentum and growth. Ironically, these new insights did not come from the disciplines traditionally concerned with mind, from philosophy or psychology. Instead, they evolved from the merger of these disciplines with the biology of the brain, a new synthesis made possible by the remarkable achievements in molecular biology. The result has been a new science of mind, a science that has harnessed the power of molecular biology to examine the great remaining mysteries of life.

\section{Mind and Brain}

This new science is grounded by five principles. First, mind and brain are inseparable. The brain is a multifaceted biological organ of vast computational abilities that constructs our sensory experiences, regulates our thoughts and emotions, and mediates our actions. Our brain is responsible not only for motor behaviors such as running and eating but also for the complex and multifaceted acts considered quintessentially human, such as thinking, speaking, and creating works of art.

Second, each mental function in the brain, from the simplest reflexes to the most creative acts in language, music, and art, is carried out by specialized neural circuits throughout different regions of the brain. It has been noted by many in the neuroscience community that it is preferable to use the term biology of mind to 
refer to the set of mental operations carried out by these specialized neural circuits rather than biology of the mind, which can be seen to inaccurately connote that there is a unique or singular place, a single location in the brain, that carries out mental operations.

Third, all of these circuits are composed of the same elementary signaling units, the neuron. Fourth, these neural circuits use specific molecules to generate signals within and between nerve cells. Finally, the specific signaling molecules have been conserved and retained through millions of years of evolution. Some of them were present in the cells of our most ancient ancestors and can be found today in our most distant and primitive evolutionary relatives.

Hence, we gain from this new knowledge regarding the science of mind not only insights into ourselveshow we perceive, learn, remember, feel, and act—but also a new viewpoint of ourselves within the context of biological evolution. Accordingly, this allows us to appreciate that the human mind evolved from molecules used by our most primitive ancestors and that the extraordinary conservation of the molecular mechanisms that regulate life's various processes also applies to our mental life.

In a similar vein, the search for EMDR's mechanisms of action began in the early 1990s, initially proceeding slowly and tentatively. As we entered the new millennium, the pace quickened. Theoretically driven speculative models, grounded in empirical findings from related neurobiological research bases, became more detailed and prevalent. In parallel, neurobiological studies became increasingly widespread, utilizing psychophysiological and neuroimaging examinations of EMDR treatment.

In the past decade, it has become increasingly apparent that people lacking a background in science are as enthusiastic to learn about this new knowledge regarding the science of mind and consciousness as scientists are to explain it.

\section{Scientific Growth of EMDR}

A similar phenomenon can be seen in the EMDR world. In the beginning, few were interested in the neurobiology of EMDR. A talk speculating on EMDR's neural mechanisms would attract 30 people, on a good day. As in other aspects of neuroscience, this interest has exploded. Hundreds are now in attendance at EMDR workshops currently held worldwide, solely focused on the topic of the neurobiology of EMDR. Hence, these occurrences have made it apparent that nonscientists are prepared to make the effort to understand the key issues of brain science if scientists are willing to make the effort to explain them.

Hence, consciousness and EMDR are inextricably intertwined, giving us an information processing paradigm that provides an integrated approach that can incorporate and interpret key aspects of such diverse modalities as psychodynamic, behavioral, cognitive, gestalt, ego-state, and body-oriented therapies. If the neurobiology of consciousness enables our understanding of the neural interrelationship between self and object, EMDR has given us both tools and mysteries to solve in the repair of the self and its relation to its objects.

\section{References}

Bergmann, U. (2012). Neurobiological foundations for EMDR practice. New York, NY: Springer Publishing.

Damasio, A. R. (1999). The feeling of what happens. New York, NY: Harcourt Brace.

Hobson, A. (2009). Prologue. In S. Laureys \& G. Tononi (Eds.), The neurology of consciousness (pp. xi-xii). New York, NY: Elsevier, Academic Press.

Kandel, E. R. (2006). In search of memory: The emergence of a new science of mind. New York, NY: W. W. Norton.

Llinas, R. R. (2001). I of the vortex: From neurons to self. Cambridge, MA: The MIT Press.

Ramon y Cajal, S. (1899). Textura del systemo nervioso del hombre $y$ de los vertebrados [Texture of the nervous system of man and vertebrates]. Madrid, Spain: Moya.

Shapiro, F. (1995). Eye movement desensitization and reprocessing: Basic principles, protocols, and procedures (1st ed.). New York, NY: Guilford Press.

Shapiro, F. (2001). Eye movement desensitization and reprocessing: Basic principles, protocols, and procedures (2nd ed.) New York, NY: Guilford Press.

Correspondence regarding this article should be directed to Uri Bergmann, 366 Veterans Memorial Highway, Suite 1A, Commack, NY 11725. E-mail: ubergmann@att.net 